endotherapy (median 2.5 treatments; range 2-12); mean SS was 1.0 at latest follow-up.

Per-procedure, mean reduction in SS was 0.8 points $(\mathrm{p}<0.01)$ with overall positive response rate of $67 \%$. By symptom, vomiting was most responsive to endotherapy $(86 \%$ pre $\mathrm{v} 32 \%$ post). By treatment type, Botox alone $(\mathrm{n} \geq 66)$ had the highest overall response $(78 \%)$ compared to $\mathrm{EBD}(38 \%$, $\mathrm{p} \geq 0.02)$ or combination therapy $(66 \%, \mathrm{p} \geq 0.3)$. Response to Botox was greater in patients under $40(83 \% \mathrm{v} 61 \%, \mathrm{p} \geq 0.04)$ and females $(81 \% \mathrm{v} 33 \%, \mathrm{p} \geq 0.002)$. By indication, diabetic $\mathrm{GP}(\mathrm{n} \geq 17)$ were most likely to respond $(76 \%)$.

Sub-group analysis showed procedures for gastroparesis (diabetic/idiopathic, $\mathrm{n} \geq 75$ ) responded significantly more to Botox (mean SS reduction 1, p<0.01) than EBD (mean SS reduction $0.2, \quad \mathrm{p}>0.1$ ) or combination therapy (mean SS reduction $0.44, \mathrm{p} \geq 0.12$ ). Procedures for gastric transposition $(n \geq 42)$ showed significant SS reduction post combination therapy $(2.1 \mathrm{v} 1.2, \mathrm{p} \geq 0.01)$ but not post $\operatorname{EBD}(1.9 \mathrm{v} 1.6, \mathrm{p}>0.1)$ or Botox (1.7 v 1.1, p $\geq 0.08)$.

Conclusions Endotherapy is a safe and effective treatment for refractory gastroparesis. We found Botox monotherapy significantly improved symptoms in diabetic or idiopathic gastroparesis, especially younger females; conversely, combination therapy was preferable for delayed gastric emptying post gastric transposition. Careful patient selection may augment therapeutic response.

\section{PWE-133 IMPACT OF THE NORTH AMERICAN CONSENSUS ON HYDROGEN AND METHANE-BASED BREATH TESTING FOR CARBOHYDRATE MALABSORPTION}

Mr Jordan Haworth*, Jennifer Haynes*, Charlotte Pitcher, Sam Treadway, Anthony Hobson. The Functional Gut Clinic, Manchester, UK

\subsection{6/gutjnl-2018-BSGAbstracts.427}

Introduction The acquisition parameters and interpretation of breath testing data for the assessment of carbohydrate malabsorption (CM) varies widely between centres. The North American Consensus (NAC) document on breath testing published in 2017 was a first attempt to standardise this diagnostic test. Two key recommendations were to extend the period of post ingestion breath sampling from 120 to $180 \mathrm{~min}$ and that SIBO should be excluded prior to CM testing. We retrospectively assessed our database of $\mathrm{CM}$ studies from the previous 12 months to examine the impact of these recommendations on results.

Methods Patient data was retrospectively attributed to $120 \mathrm{~min}$ and $180 \mathrm{~min}$ groups for both lactose and fructose breath tests. All patients provided a baseline sample prior to ingestion of $25 \mathrm{~g}$ of either substrate following a 24 hour restrictive diet and 12 hour fast. In total 200 breath tests were analysed (120-lactose and 80 -fructose). A rise $>20 \mathrm{ppm}$ above baseline was considered positive for CM. The results were compared statistically using Pearson's chi-squared test.

Results A positive result for CM at $120 \mathrm{~min}$ was seen in 27 of $120(22.5 \%)$ subjects for lactose and 33 of $80(36.3 \%)$ subjects for fructose. When extended to $180 \mathrm{~min}$ the number of positive CM tests increased to $30 \%$ and $41.3 \%$ for lactose and fructose, respectively. Within these sub-groups the significant rise in gas levels occurred at $\leq 60 \mathrm{~min}$ after ingestion in $34.3 \%$ for lactose and $69.8 \%$ for fructose. There was a significant association between patients who had a positive SIBO test (as determined by a separate lactulose test) and a positive breath test for lactose at $\leq 60 \mathrm{~min}\left[\chi^{2} \geq 5.3, p 0.02\right]$. Findings for fructose were not significant $[p>0.05]$.

Conclusions Around $20 \%$ of the positive results for CM occurred after $120 \mathrm{~min}$ supporting the NAC position to extend the post ingestion period to $180 \mathrm{~min}$ to avoid false negative studies. SIBO may influence results and a lactulose breath test should be performed prior to $\mathrm{CM}$ testing to avoid false positive tests and to help interpret $\mathrm{CM}$ studies with greater accuracy. Like the first iteration of the Chicago Classification for oesophageal motility testing - the NAC on breath testing represents a positive first step in standardising these diagnostic tests.

\section{PWE-134 MANAGEMENT OF GASTROPARESIS: CURRENT PRACTICE IN A TERTIARY CENTRE}

Sailish Honap*, Miriam Dixon-Zegeye, Fatima Olivares, Giovanni Tritto. Guy's And St. Thomas' NHS Foundation Trust, London, UK

\subsection{6/gutjnl-2018-BSGAbstracts.428}

Introduction Gastroparesis is a syndrome characterised by delayed gastric emptying in the absence of mechanical obstruction. The aim of this study was to assess consistency and adherence to guidelines of current practice and to evaluate the effectiveness of routinely implemented interventions in a large London tertiary centre.

Methods A retrospective study was conducted by examining records of all adult patients with delayed gastric emptying, objectively measured by NM scintigraphy, between 20102017. Effectiveness was defined as evidence of symptomatic improvement either semi-quantitatively by the Gastroparesis Cardinal Symptom Index (GCSI) or by documented qualitative evidence from clinical records, before and after intervention. Our practice was compared to recommendations published by the American College of Gastroenterology in 2013.

Results We identified 91 patients diagnosed with gastroparesis from 655 consecutive scans. Of these, 46 were excluded due to incomplete records. 55 patients were included: median age 48 (range 21-89), 67\% female. Diabetes (40\%) was the commonest cause; $40 \%$ of cases were idiopathic.

Conservative management $34 / 55(62 \%)$ patients had dietetic input with $16 \%$ requiring enteral nutrition. $17 / 55$ (31\%) of patients were taking a drug known to delay gastric emptying but stopped in only $12 \%$ of patients.

Medical management 48/55 (87\%) patients received prokinetics, including metoclopramide 28/55 (51\%), domperidone $33 / 55(60 \%)$ and erythromycin 19/55 (35\%) with treatment duration specified in only $30 \%$. No patients had documented GCSI. From qualitative records, $7 / 48$ (15\%) of these reported some benefit, while 20/48 (42\%) had no effect and in $21 / 48$ (44\%) the effect was unknown.

Intrapyloric Botox was administered in 25/55 (45\%) of patients but results were not documented by GCSI. Nevertheless, 11/25 (44\%) of patients reported some benefit. Additionally, 2 patients had pyloric dilatation and 1 feeding jejunostomy and venting gastrostomy.

Conclusions The management of gastroparesis showed wide variations in practice in our institution. The lack of semiquantitative assessment of the results of different interventions hindered evaluation of effectiveness. Conservative measures, including discontinuation of contributing drugs, were adopted 
only in a minority of patients. Prokinetics are widely used as first choice approach but treatment duration and stopping rules were not clearly established. Intrapyloric Botox injection showed subjective benefit in a proportion of patients, matching results of previous RCTs where placebo had similar benefit. Our study indicates the need for a more consistent and evidence-based management of gastroparesis. Dedicated outpatient clinics and internal protocols may help to achieve this task.

\section{PWE-135 CONSTIPATION IS THE MOST COMMON GASTROINTESTINAL SYMPTOM IN ACROMEGALY}

Nashiz Inayet*, Jamal Hayat, Gul Bano, Andrew Poullis. St George's Hospital and St George's, University of London, London, UK

\subsection{6/gutjnl-2018-BSGAbstracts.429}

Introduction Acromegaly is caused by a pituitary somatotroph adenoma resulting in excess secretion of growth hormone which leads to excess secretion of Insulin like growth factor 1 from the liver, causing abnormal soft tissue growth with a variety of symptoms and an increased risk of colorectal cancer. Somatostatin analogues, used in the management of acromegaly are also associated with a range of abdominal symptoms. We collected data from patients with a confirmed diagnosis of acromegaly to evaluate the frequency, type and burden of abdominal symptoms.

Methods Data was collected from patients with confirmed Acromegaly using SF36 RAND and Rome IV Diagnostic questionnaire and compared to a control group, to assess the burden of GI symptoms. Data analysis was carried out using Microsoft Excel and IBM SPSS v 25.

Results 49 acromegalics (23 male and 26 females; age range 23-64 years, mean 43) and 200 controls (92 male and 108 females; age range $18-84$, mean 42.4) were recruited. $94 \%$ (46 out of 49) of acromegalics reported abdominal symptoms and $79 \%$ (39 out of 49) had at least one FGID according to the Rome IV diagnostic criteria, compared to $36 \%$ of controls $(\mathrm{OR}>1, \mathrm{p}<0.0001)$. More acromegalic females reported suffering from abdominal symptoms (100\%) than males (87\%), however there was no significant difference observed in the frequency and intensity of symptoms. The most commonly reported symptom was constipation $(69 \%$ acromegalics vs $21 \%$ of controls OR $>1, \mathrm{p}<0.0001,95 \% \mathrm{CI} 4.4-15.8)$ followed by diarrhoea $(52 \%$ acromegalics vs $18 \%$ of controls OR $>1, \quad p<0.0001,95 \%$ CI $2.5-9.3) .30$ out of 49 (61\%) respondents met the criteria for Functional Constipation according to Rome IV. The prevalence of constipation increased with increasing age and was often associated with bloating. No statistically significant difference seen in the prevalence of upper GI, biliary and anorectal symptoms between the acromegaly patients and controls. Acromegalics scored lower on the mean scores of the eight parameters (physical functioning, role limitations due to physical health, role limitations due to emotional health, energy/fatigue, emotional wellbeing, social functioning, pain, general health (mean scores 60.04 vs 71.23 , 95\% CI -13.6829 to -8.6971 , OR $>1, p$ value $<0.001)$ measured by the RAND SF36 as compared to the control group.

Conclusions The presence of FGID affecting the lower gastrointestinal tract in acromegalics is substantially higher than the controls in our study. Factors such as delayed transit and increased bowel length may play a role. Functional Constipation is the most commonly reported problem. Poorer quality of life may in part be attributable to abdominal symptoms. Symptoms of the upper GI tract such as reflux, dyspepsia and vomiting do not appear to occur more frequently in this group as compared to controls.

\section{PWE-136 FUNCTIONAL GASTROINTESTINAL DISORDERS (FGID) IN EHLERS DANLOS TYPE III (HYPERMOBILE) AND MARFAN SYNDROME PATIENTS}

Nashiz Inayet*, Jamal Hayat, Maite Tome, Arvind Kaul, Ann Child, Andrew Poullis. St George's, University of London, London, UK

\subsection{6/gutjnl-2018-BSGAbstracts.430}

Introduction Ehlers Danlos syndrome is a group of inherited heterogenous multisystem disorders characterised by skin hyperextensibility, atrophic scarring, joint hypermobility and generalised tissue fragility. Hypermobile EDS (hEDS)is the most common type. Marfan syndrome(MS) is also a multisystem disorder caused by a mutation in FBN1 gene which shares some phenotypic features with Hypermobile EDS such as joint hypermobility. Recent studies have suggested an association of Functional Gastrointestinal Disorders with joint hypermobility.

Methods Data was collected from 27 MS patients (10 male and 17 females, age range 19-35 years mean 27) and 33 hEDS patients (3 male and 30 females, age range 19-32 years mean 23) with no organic gastrointestinal diagnosis, using SF36 RAND and Rome IV Diagnostic questionnaire and compared to control group (200 respondents, 92 male and 108 female; age range $18-84$, mean 42.4 ) to assess the burden of GI symptoms in these patients. Data analysis was carried out using Microsoft Excel and SPSS version 25 (IBM Corporation, America).

Results In both groups the majority $(78.3 \%)$ of respondents were female within the age range of 19-35 years. Both groups of patients showed a higher prevalence of abdominal symptoms as compared to the control group, however the hEDS group not only showed a higher prevalence but more frequent and severe symptoms meeting Rome IV criteria for diagnosis of FGIDs. 16 (49\%)of the EDS patients met the criteria for more than one FGID.

$\mathrm{p}$ values were significant $(\mathrm{p}<0.001)$ for functional heartburn, functional dyspepsia, functional dysphagia, IBS-D and functional bloating in hEDS patients when comparing the prevalence to controls.

The hEDS group also scored lower on quality of life scores (QOL) in comparison to either of the other groups with a mean score of 48.6 (95\%CI 25.3-33.4,p $<0.0001)$ as compared $54.2(95 \%$ CI $20.9-29.0, \mathrm{p}<0.0001)$ in the Marfan group and 78.6 in the control group.

Conclusions FGIDs are reported in both Marfan syndrome and Hypermobile Ehlers Danlos syndrome but appear to be more common and severe in hEDS. These patients score lower on quality of life scores as well despite hypermobility being a common feature of both conditions. Further research is needed in this area to see whether there are other factors that can explain this difference. 